\title{
Confectionery Xylitol Gum-Containing Tablets for Medical Application and the Sintering Effect on Gum Tablets
}

\author{
Tatsuaki Tagami, Eriko Kuwata, and Tetsuya Ozeki* \\ Drug Delivery and Nano Pharmaceutics, Graduate School of Pharmaceutical Sciences, Nagoya City University; \\ 3-1 Tanabe-dori, Mizuho-ku, Nagoya 467-8603, Japan. \\ Received April 13, 2021; accepted June 13, 2021
}

\begin{abstract}
Confectionery ingredients are expected to enhance the medication adherence of pediatric patients taking bitter-tasting drugs when adequate pediatric medicines are not available in practical settings. Gum is a familiar confectionery, and several drug-loaded gums are on the market as medicated chewing gums. In this study, medical gum tablets composed of confectionery xylitol gum and a drug (ibuprofen or acetaminophen) were prepared and evaluated for the purpose of potential hospital applications. The effect of the sintering process, a heating treatment, on the physical properties of the solid materials was also examined. The sintering process markedly improved the hardness of the gum tablets. The sintering temperature and time affected the hardness of both ibuprofen- and acetaminophen-loaded gum tablets, whereas heat treatment around the melting point of ibuprofen or xylitol and longer heat treatment resulted in failure of the preparation or a reduction in hardness. The sintered gum tablets exhibited a delayed drug release profile in artificial saliva after an in vitro chewing test. The current results provide basic and useful information about the preparation of gum-containing tablets in future clinical settings.
\end{abstract}

Key words confectioneries ingredient; hospital preparation; medicated chewing gum (MCG); sintering; tablet; xylitol gum

\section{INTRODUCTION}

Medicated chewing gums (MCGs), oro-mucosal formulations listed in the United States Pharmacopeia (USP), European Pharmacopoeia (EP), and Japanese Pharmacopoeia (JP), have received increased consideration from pharmaceutical companies due to various potential advantages. ${ }^{1-3)}$ MCGs can be utilized to enhance drug absorption from the oral mucosa into blood circulation. For example, nicotine-loaded gum (Nicotine polacrilex, Nicorette) for nicotine replacement therapy in patients undergoing smoking cessation treatment is familiar, with other dosage forms available as over-the-counter drugs. ${ }^{4,5)}$ After initial chewing by patients, the nicotine-loaded gum is parked between the cheek and gum to allow the buccal mucosa to absorb the nicotine. Absorption of the drug via the buccal mucosa can prevent the first-pass effect that occurs in the liver. Repeated cycles of chewing and parking allow for slow and intermittent drug absorption. Chewing nicotine gums has the effect of satisfying and distracting patients from their nicotine craving.

MCGs containing other drugs have also been developed for systemic use (e.g., dimenhydrinate, ${ }^{6)}$ fenoprofen, ${ }^{7)}$ caffeine, ${ }^{8)}$ and insulin $\left.{ }^{9}\right)$. Other applications of gum-based drugs in addition to their use in oro-mucosal formulations have also been investigated. For example, gum formulations can be used for localized drug release in the oral cavity to suppress caries development, plaque formation, oral candidosis, lichen planus, and mouth sores caused by cancer therapy (antimicrobial peptides,${ }^{10)}$ green tea, ${ }^{11)}$ vitamin $\mathrm{C},{ }^{12)}$ chlorhexidine, ${ }^{13,14)}$ miconazole ${ }^{15)}$ pycnogenol, ${ }^{16)}$ aloe vera ${ }^{17)}$ ). However, despite their usefulness, the number of MCGs on the worldwide market is still low. The development of new gum formulations that take advantage of the unique characteristics of gum is thus expected.

The use of confectionery ingredients in drug formulations has been studied as a means of improving patient adherence to drug regimens. The pleasant texture and flavor of these ingredients can improve the acceptability of medicines to patients, especially pediatric patients. If there is not an appropriate medicine for a pediatric patient, tablets or capsules are ground or disrupted, and then liquid or powder formulations are prepared by pharmacists in accordance with the situation. ${ }^{18)}$ In order to alleviate bitter tastes, the ground or disrupted powder is sometimes mixed with juice or ice cream, as there may not be an adequate and satisfactory drug formulation for these patients. The replacement of conventional drug formulations with confectionery ingredients may be useful in hospital practice. For instance, several drug formulations containing confectionery ingredients have been reported for use in pediatric patients (e.g., "gummy" formulations ${ }^{19,20)}$ and chocolate formulations $\mathrm{s}^{21,22}$ ).

In this study, we focused on medicinal gum tablets containing active pharmaceutical ingredients (APIs) and confectionery xylitol gum as a new platform for hospital preparation by pharmacists. The incorporation of confectionery xylitol gum into a drug tablet has several potential advantages. The gum tablets have the effect of preventing dental caries due to the xylitol-based gum. Additionally, gum tablets often mask the bitter taste of APIs due to the presence of strong sweeteners in the xylitol gum. Although the base of confectionery gum can contain multiple complex ingredients (e.g., polyvinyl acetate, ester gum, styrene-butadiene rubber, paraffin wax), the texture of commercially available gums has been well-optimized for consumers, although formulating them with APIs requires specialized expertise. These types of gum tablets can be patient friendly, thus improving drug adherence. However, to 
the best of our knowledge, the physical properties of confectionery gum tablets containing APIs have not been thoroughly investigated. The purpose of this study therefore was to investigate how the heat treatment process of sintering affects various properties of gum tablets. Sintering is used in pottery and ceramics making to reinforce their firmness, traditionally because heating a solid material below its melting point reduces its porosity by plastic deformation of the particles in the material. In the pharmaceutical field, some studies have reported that the sintering process affects the breaking force of tablets containing polymers ${ }^{23)}$ and drug release from polymer matrix tablets $^{24)}$ and pellets. ${ }^{25)}$ In addition, sintering contributes to the formation of the polymer coating of microparticles, known as the curing process. ${ }^{26)}$ The sintering technique is also used to promote the self-healing process of porous polymer microparticles so that they will encapsulate macromolecules for drug delivery. ${ }^{27)}$ For these reasons, in this study, we investigated the effect of the sintering process on the physical properties and drug release of novel gum-based tablets.

\section{MATERIALS AND METHODS}

Materials Xylitol gum (ORATECT GUM) was purchased from LOTTE Co., Ltd. (Tokyo, Japan). According to the manufacturer, the xylitol gum contains xylitol (40\%), gum base, maltitol, eucalyptus extract, acesulfame K, sucralose, Arabic gum, gelatin, hesperidin, titanium dioxide, brightener, and flavoring. Acetaminophen was purchased from Tokyo Chemical Industry Co., Ltd. (Tokyo, Japan). Ibuprofen and xylitol were purchased from FUJIFILM Wako Pure Chemical Corporation (Osaka, Japan).

Preparation of Gum Tablets As a typical experiment, several xylitol gum samples were pulverized for 2 min using an electric coffee grinder. Ibuprofen or acetaminophen was then mixed with the pulverized xylitol gum at a ratio of $1: 2$ or $1: 5$ (drug/gum, w/w). The median diameter of both ibuprofenloaded and acetaminophen-loaded gum powder particles was approximately $40 \mu \mathrm{m}$, as determined from observation of particles $(n \approx 100)$ using scanning electron microscopy (S-4300; Hitachi, Tokyo, Japan). The powder mixture $(300 \mathrm{mg})$ was loaded into round concave dies, and corresponding punches were set. The gum tablets were compressed using a manual hydraulic press at various pressures (490, 980, and 1960N). The resulting tablets (diameter: $10 \mathrm{~mm}$ ) were then evaluated in subsequent experiments.

Sintering Process Tablets prepared as described in the previous section were placed on a stainless steel plate, and the plate was then placed on the shelf of an electric drying oven (DRN320DD; Advantec Toyo Kaisha, Ltd., Tokyo, Japan) maintained at a pre-determined temperature. At various time points, the plate was taken from the oven, and the samples were allowed to cool to room temperature.

Hardness Test The hardness of the gum tablets was measured using a Kiya hardness tester (Kiya Seisakusho, Tokyo, Japan).

Powder X-Ray Diffraction (PXRD) PXRD spectra were recorded on a MiniFlex600 instrument (Rigaku Co., Tokyo, Japan). The tube voltage and tube current were $40 \mathrm{kV}$ and $15 \mathrm{~mA}$, respectively, and the PXRD data were recorded between 5 and $33^{\circ}$. Regarding samples of xylitol gum, the coating was peeled off to separate the coating materials and inner contents of the gum, which were analyzed separately. Ibuprofen- and acetaminophen-loaded tablets were pulverized using an electric coffee grinder, and the resulting samples were used for measurements.

Differential Scanning Calorimetry (DSC) Samples of approximately $2-3 \mathrm{mg}$ were placed in aluminum pans and sealed. DSC thermograms of samples were obtained using a DSC-60 instrument (Shimadzu Corporation, Kyoto, Japan) under purging with $\mathrm{N}_{2}$ gas. The temperature range was $30-300^{\circ} \mathrm{C}$. Samples of gum coating material and inner contents were prepared as described in "Powder X-Ray Diffraction (PXRD)" above. For heat-treated and untreated drug-loaded tablets, the pulverized samples were used for measurements.

In Vitro Chewing Test (Drug Release Test) Drug release tests were conducted by partially imitating the chewing of an MCG formulation. First, artificial saliva ( $\mathrm{pH} 7.0$; $1.5 \mathrm{mM} \mathrm{CaCl}_{2}, 3.6 \mathrm{mM} \mathrm{K \textrm {K } _ { 2 }} \mathrm{PO}_{4}, 100 \mathrm{mM} \mathrm{KCl}$, and $20 \mathrm{mM}$ 4-[2-hydroxyethyl]-1-piperazineethanesulfonic acid, titrated with $\mathrm{NaOH}$ ) was prepared according to a previous report, with modifications. ${ }^{28)}$ Next, $20 \mathrm{~mL}$ of artificial saliva was poured into a small plastic cup, and a gum tablet was placed in the cup. The gum tablet was manually pressed using a plastic stick (diameter $1.5 \mathrm{~cm}$ ) every $2 \mathrm{~s}$. In advance, the experimenter was trained to produce a relatively stable pushing force $(\approx 5 \mathrm{kgf})$ using a weighing scale. The gum tablet was pressed for $30 \mathrm{~s}$, and the sample was put aside for $1 \mathrm{~min}$ as an interval. This "pressing and put-aside" process was repeated 7 times. At each interval, $100 \mu \mathrm{L}$ of sample was removed and replaced with an equal volume of artificial saliva. The absorbance of the sample solution was measured using a UV-visible spectrophotometer (UV-1800, Shimadzu: ibuprofen, $263 \mathrm{~nm}$; acetaminophen, $243 \mathrm{~nm}$ ). Linear standard curves of drug concentration versus absorbance were prepared from analysis of solutions containing drug and gum powder because the gum formulations could interfere the absorbance of the drug.

Statistical Analysis Basic statistical parameters were determined using Microsoft Excel functions. Initially, $F$ tests were performed to assess the equality of variance between data from two groups. Student's $t$ tests were conducted when the null hypothesis regarding homoscedasticity was not rejected. In contrast, Welch's $t$ tests were conducted when the alternative hypothesis regarding heteroscedasticity was adopted. Statistical significance was defined as a $p$-value $<0.05$.

\section{RESULTS AND DISCUSSION}

Effect of the Sintering Process on the Hardness of Gum Tablets In the current study, we examined the effect of sintering on xylitol gum-containing tablets. Two different antipyretic analgesics (ibuprofen and acetaminophen) were used as model drugs for the gum tablets because these drugs are widely utilized for treating not only systemic inflammation but also local inflammation in the oral cavity. ${ }^{29,30)}$ To the best of our knowledge, there are no previous reports regarding the sintering of gum-based medical tablets. The glass transition temperature of polyvinyl acetate incorporated in typical chewing gum is approximately $31^{\circ} \mathrm{C},{ }^{31)}$ so that the gum base is designed to soften near the normal temperature of the oral cavity. The sintering process is applied above the glass transition temperature, so we conducted heat treatment of gum tablets in the range $40-90^{\circ} \mathrm{C}$. 


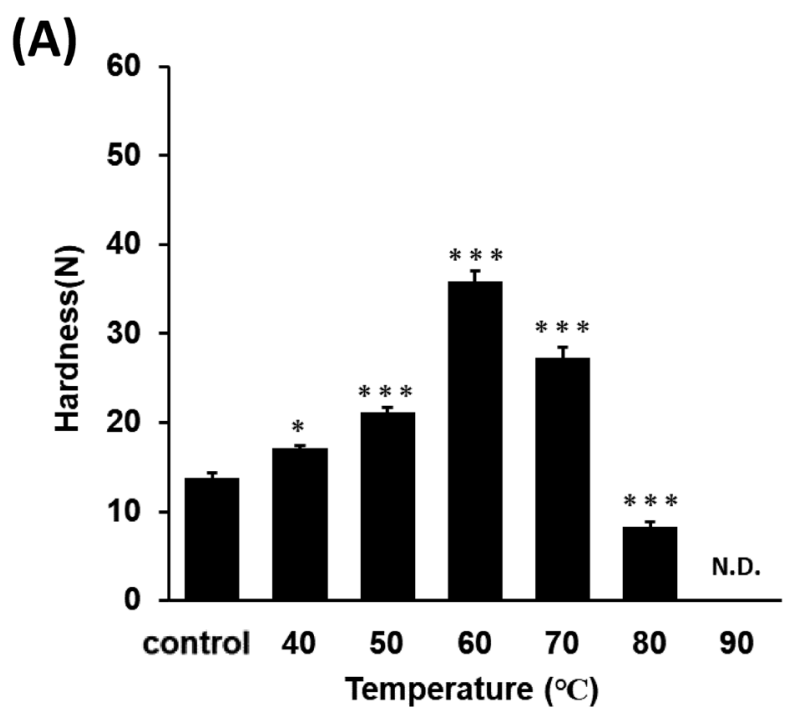

(B)
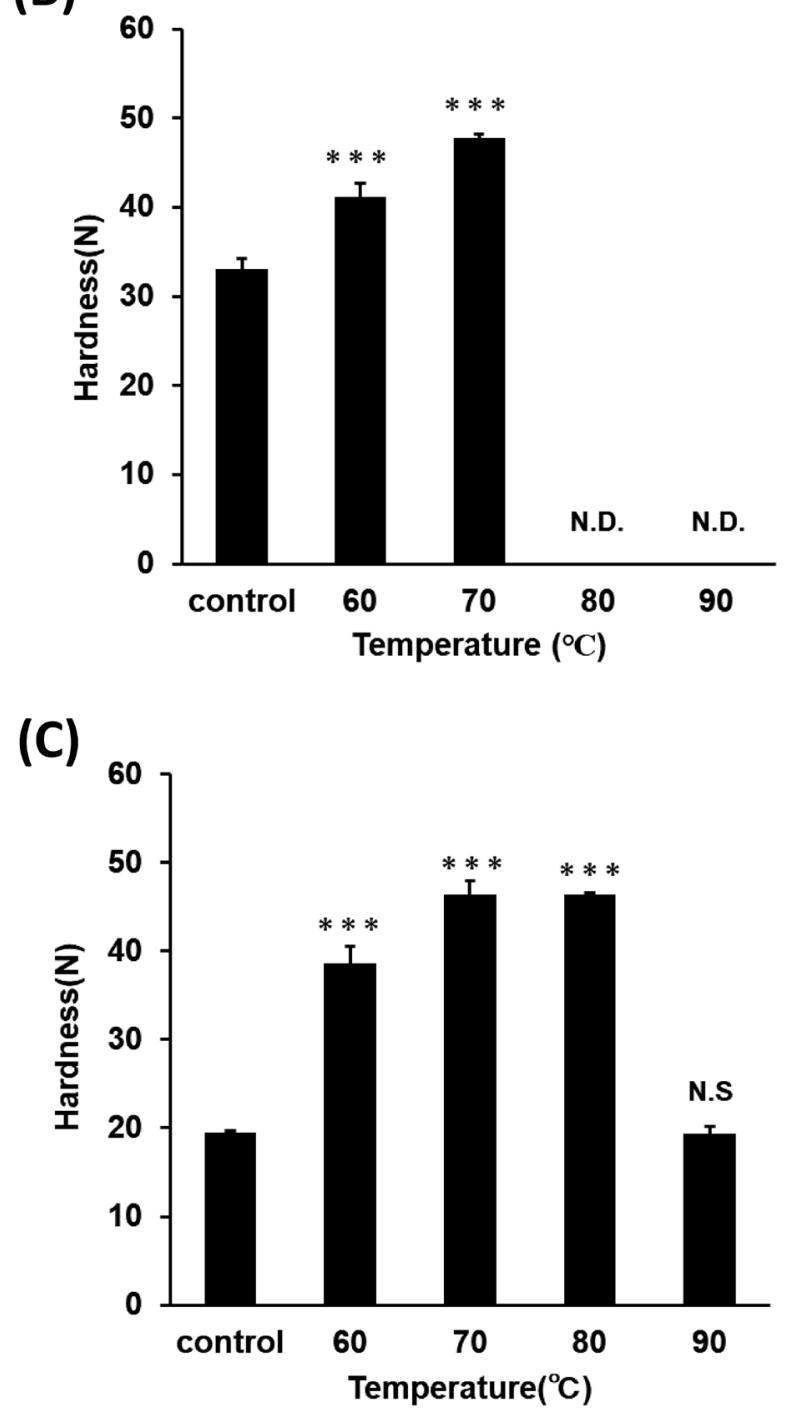

Fig. 1. Effect of Sintering Temperature on the Hardness of Gum Tablets

(A) Gum tablets without drugs, (B) ibuprofen-loaded gum tablets, and (C) acetaminophen-loaded gum tablets. Each tablet was incubated at a different temperature for $15 \mathrm{~min}$. Data represent the mean \pm standard deviation (S.D.) $(n=5)$. Significant differences versus the control are indicated as follows: *, $p<0.05$; ***, $p<0.005$; N.S.; not significant. N.D.; not determined due to leakage of liquid from tablets.

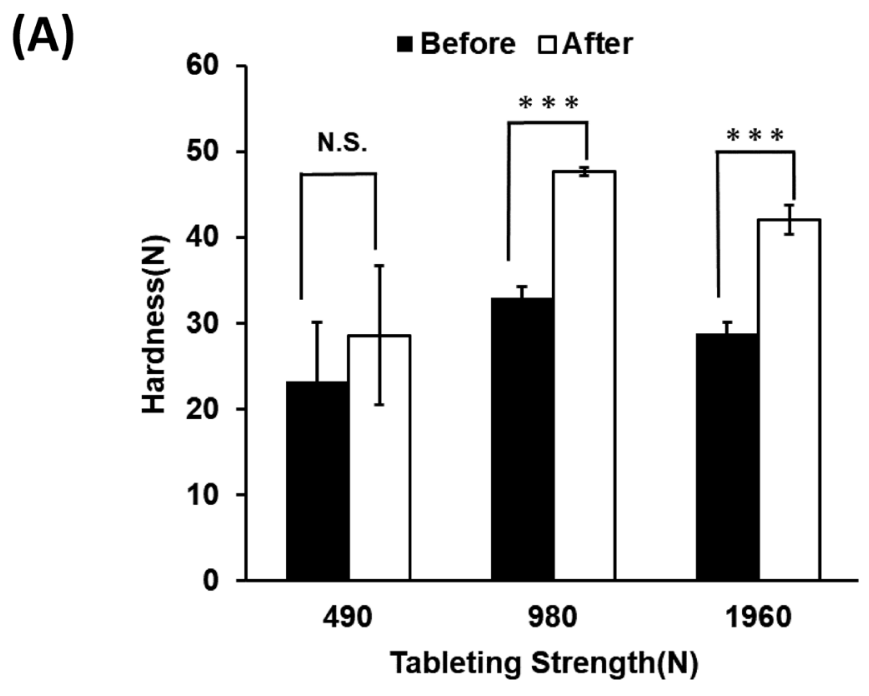

(B)

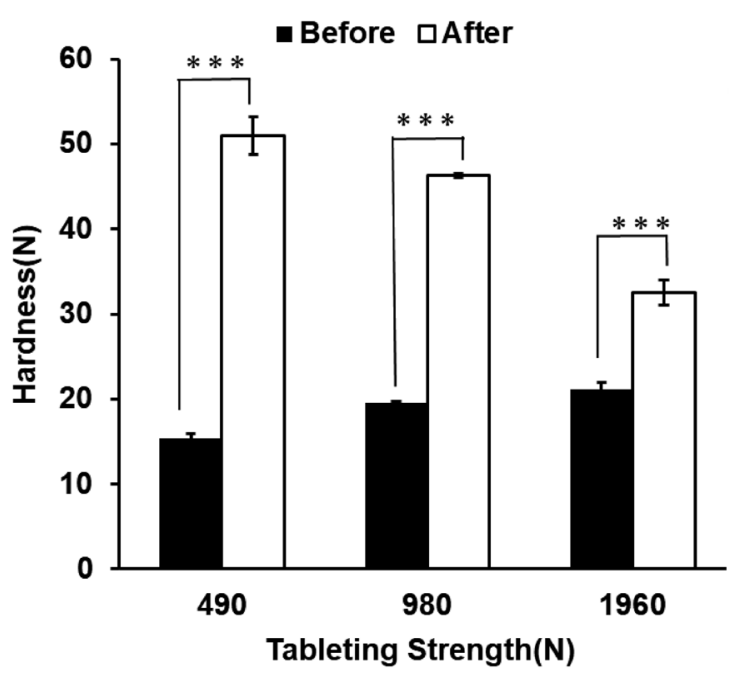

Fig. 2. Effect of Sintering on the Hardness of Drug-Loaded Tablets at Different Tableting Strengths

(A) Ibuprofen-loaded gum tablets and (B) acetaminophen-loaded gum tablets. Ibuprofen-loaded tablets or acetaminophen-loaded tablets were incubated at $70^{\circ} \mathrm{C}$ for $15 \mathrm{~min}$ or $80^{\circ} \mathrm{C}$ for $15 \mathrm{~min}$, respectively. Data represent the mean \pm S.D. $(n=5)$. Significant differences versus the control are indicated as follows: $* * * ; p<0.005$; N.S.; not significant.

Gum tablets with and without drugs were heated for a short time $(15 \mathrm{~min})$ at different temperatures, and the hardness of the tablets was then evaluated (Fig. 1). As shown in Fig. 1A, the null gum tablets (without drug) exhibited a slight but significant increase in hardness at 40 and $50^{\circ} \mathrm{C}$, and the hardness was highest at $60^{\circ} \mathrm{C}$ and then decreased at 70 and $80^{\circ} \mathrm{C}$. Leakage of liquid from the tablets was observed at $90^{\circ} \mathrm{C}$, likely because the temperature was near the melting point of xylitol $\left(92-96^{\circ} \mathrm{C}^{32}\right)$. The hardness of ibuprofen- and acetaminophen-loaded tablets was significantly increased at 60 and $70^{\circ} \mathrm{C}$ (for ibuprofen-loaded tablets) and $60-80^{\circ} \mathrm{C}$ (for acetaminophenloaded tablets) (Figs. 1B, C). Heat treatment at higher temperatures induced leakage of liquid from the tablets $\left(>80^{\circ} \mathrm{C}\right.$, ibuprofen-loaded tablets; $90^{\circ} \mathrm{C}$, acetaminophen-loaded tablets). This was probably due to the melted ibuprofen (melting point, $75-78^{\circ} \mathrm{C}^{33)}$ ) and xylitol (melting point, $92-96^{\circ} \mathrm{C}^{32)}$ ). It is worth noting that the order of hardness was ibuprofen-loaded gum tablets $>$ acetaminophen-loaded tablets $>$ null tablets, as the 
hardness of gum tablets at room temperature (control) showed (Figs. 1A-C). Under the present experimental conditions, hardness increased with increasing amount of API.

Next, drug-loaded gum tablets were prepared at different tableting strengths, and the effect of short-duration heating on the hardness of the tablets was investigated (Fig. 2). The hardness of gum tablets without heat treatment was similar to that of ibuprofen-loaded tablets (Fig. 2A, black bars) or slightly greater than that of acetaminophen-loaded tablets (Fig. 2B, black bars) because the tableting strength may have been relatively low in the present study compared to tablets prepared under typical conditions (e.g., $10 \mathrm{kN})$. The hardness of both ibuprofen-loaded and acetaminophen-loaded gum tablets increased significantly after heat treatment $\left(70^{\circ} \mathrm{C}\right.$ for $15 \mathrm{~min}$, ibuprofen-loaded tablets; $80^{\circ} \mathrm{C}$ for $15 \mathrm{~min}$, acetaminophen-loaded tablets), whereas ibuprofen-loaded gum tablets prepared at low tableting strength $(490 \mathrm{~N})$ did not exhibit a significant increase in hardness.

We also evaluated the hardness of drug-loaded gum tablets after heat treatment for different incubation times. As shown in Fig. 3, an increase of hardness was observed with $15 \mathrm{~min}$

\section{(A)}

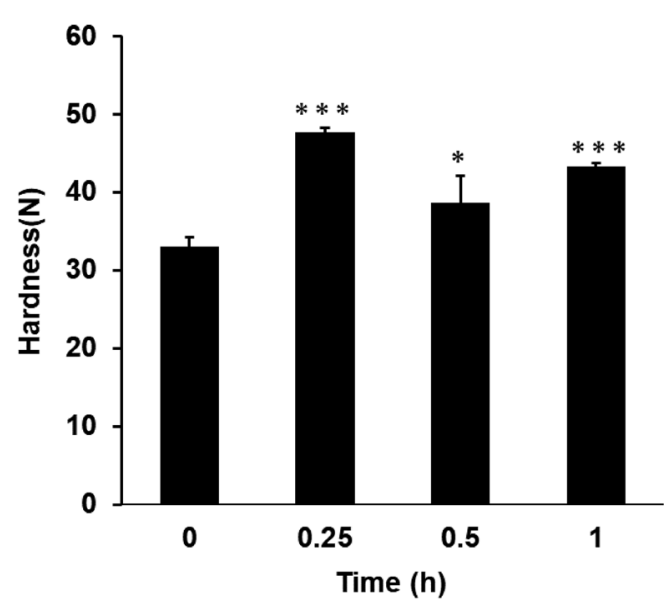

(B)

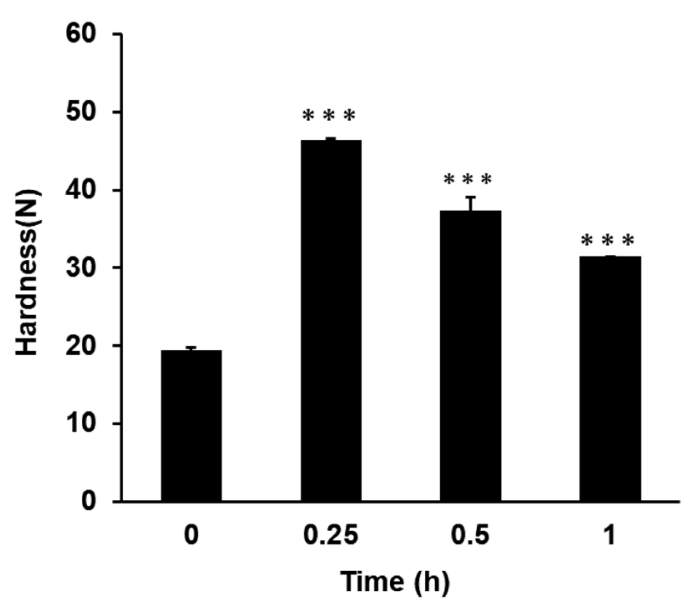

Fig. 3. Effect of Sintering Time on the Hardness of Drug-Loaded Gum Tablets

(A) Ibuprofen-loaded gum tablets and (B) acetaminophen-loaded gum tablets. Ibuprofen-loaded tablets or acetaminophen-loaded tablets were incubated at $70^{\circ} \mathrm{C}$ for $15 \mathrm{~min}$ or $80^{\circ} \mathrm{C}$ for $15 \mathrm{~min}$, respectively. Data represent the mean \pm S.D. $(n=5)$ Significant differences versus the control are indicated as follows: *, $p<0.05$; ***; $p<0.005$. of heat treatment for both ibuprofen- and acetaminophenloaded tablets. The hardness of ibuprofen-loaded gum tablets was similar or slightly lower with increasing heat treatment duration, whereas that of acetaminophen-loaded tablets decreased markedly with increasing heat treatment duration. The current results differed from those of previous studies using sintered matrix tablets. For example, Panicker et al. showed that increased sintering time contributed the firmness of Eudragit-based tablets containing metformin. ${ }^{34)} \mathrm{We}$ guess that over-sintering may decrease the hardness of gum tablets. Over-sintering can lead to brittleness of materials due to changes in their microstructure. Chen et al. reported that sintering samples at higher temperatures induces excess grain growth of dental ceramic, resulting in a decrease in flexural strength. ${ }^{35)}$

Physical Properties of Ibuprofen-Loaded and Acetaminophen-Loaded Gum Tablets PXRD results are shown in Fig. 4. Xylitol gum had a specific peak $\left(\right.$ e.g. $\left.2 \theta=9.3^{\circ}\right)$ and partially matched the xylitol $\left(2 \theta=19.7,30.2,31.6^{\circ}\right)$. The gum coating had a specific peak of maltitol $\left(2 \theta=13.3^{\circ}\right)$, as reported in the literature. ${ }^{36)}$ The samples of drug-loaded tablets exhibited a pattern similar to that of xylitol gum, whereas peaks derived from ibuprofen $\left(2 \theta=6.0^{\circ}\right)$ or acetaminophen $\left(2 \theta=15.5^{\circ}\right)$ were observed in samples of drug-loaded tablets. Samples of heated ibuprofen- and acetaminophen-loaded tablets exhibited similar PXRD patterns compared to tablets without heat treatment. In summary, these PXRD results suggest that heat treatment below the melting points of ibuprofen and xylitol does not affect the crystalline state.

The physical properties of gum tablets and their ingredients were investigated by generating DSC curves (Fig. 5). Samples of the inner contents of xylitol gum, the main component of xylitol gum, exhibited a sharp endothermic peak at approximately $95^{\circ} \mathrm{C}$. The commercially available xylitol gum contained $40 \%$ xylitol, so these results indicate that the peak of gum alone was that of xylitol $\left(92-96^{\circ} \mathrm{C}^{32)}\right)$. We then carefully peeled off the coating of the xylitol gum and analyzed a small portion of the gum coating. An endothermic peak was observed at $145^{\circ} \mathrm{C}$, which we attributed to maltitol based on the literature $\left(149^{\circ} \mathrm{C}^{37)}\right)$. The results of PXRD analysis of the coating samples supported the current results. The peak from maltitol disappeared in the drug-loaded tablets. We hypothesize that the proportion of coating on the gum was low and that the peak may have been masked. The sample of ibuprofen-loaded gum exhibited two endothermic peaks. One peak, which appeared at approximately $75^{\circ} \mathrm{C}$, matched the peak derived from ibuprofen ${ }^{33)}$; another peak, which appeared at approximately around $94{ }^{\circ} \mathrm{C}$, matched the peak from xylitol. Unexpectedly, no peak derived from acetaminophen $\left(171^{\circ} \mathrm{C}\right)$ was observed in the samples of acetaminophen-loaded gum tablets. We consider that the peak may be masked due to the small proportion. Another possibility for this result is that xylitol begins to melt at approximately $95^{\circ} \mathrm{C}$, as mentioned above, and a large amount of melted xylitol might solubilize or interact acetaminophen crystals and cause the associated peak to disappear.

The drug contents before and after sintering process was not investigated in the current study, we guess that the influence of sintering on drug content is minimum except for the condition in which the leakage from tablets was found at higher temperature. The color change of acetaminophen was 


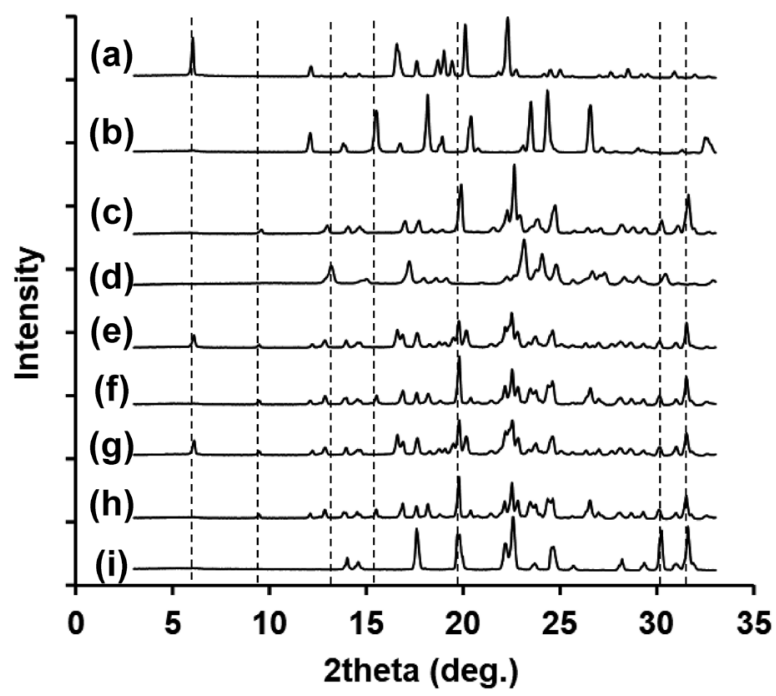

Fig. 4. PXRD Patterns of (a) Ibuprofen, (b) Acetaminophen, (c) Xylitol Gum, (d) Coating of Xylitol Gum, (e) Pulverized Ibuprofen-Loaded Gum Tablets, (f) Pulverized Acetaminophen-Loaded Gum Tablets, (g) Pulverized Ibuprofen-Loaded Gum Tablets Heated, $\left(70^{\circ} \mathrm{C}, 15 \mathrm{~min}\right)$, (h) Pulverized Acetaminophen-Loaded Gum Tablets Heated $\left(80^{\circ} \mathrm{C}, 15 \mathrm{~min}\right)$, and (i) Xylitol

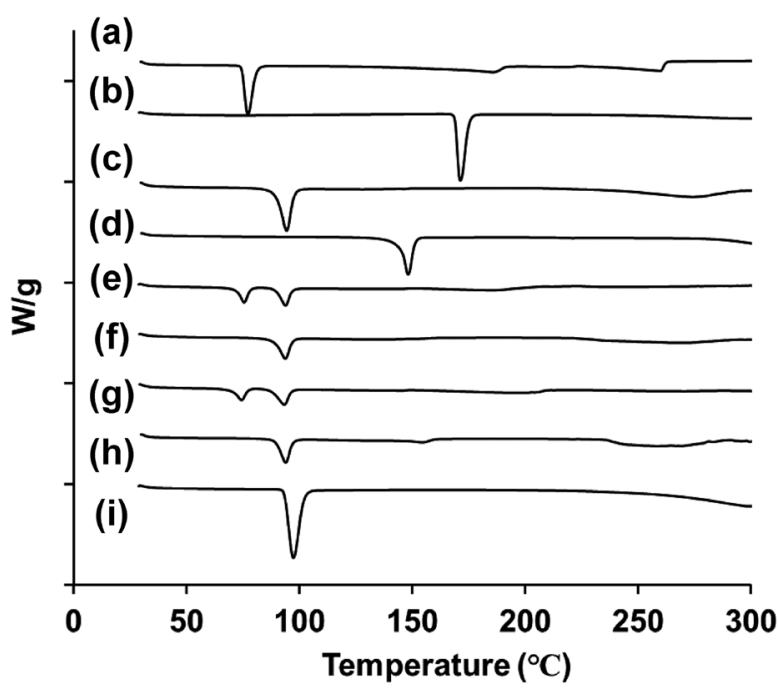

Fig. 5. DSC Thermograms of (a) Ibuprofen, (b) Acetaminophen, (c) Xylitol Gum, (d) Coating of Xylitol Gum, (e) Pulverized IbuprofenLoaded Gum Tablets, (f) Pulverized Acetaminophen-Loaded Gum Tablets, (g) Pulverized Ibuprofen-Loaded Gum Tablets Heated, $\left(70^{\circ} \mathrm{C}\right.$, $15 \mathrm{~min}$ ), (h) Pulverized Acetaminophen-Loaded Gum Tablets Heated $\left(80^{\circ} \mathrm{C}, 15 \mathrm{~min}\right)$, and (i) Xylitol

reported when it was heated by hot melt extrusion method at more than $150{ }^{\circ} \mathrm{C}^{38)}$ In our case, we did not find such a color change after sintering process, because sintering was conducted at the temperature enough below melting point $\left(80^{\circ} \mathrm{C}\right)$. That is why we consider that the influence of sintering on the acetaminophen content is minimum. Regarding ibuprofen, one report mentioned the accelerated degradation of ibuprofen when the ibuprofen-loaded tablets were incubated at $70^{\circ} \mathrm{C} / 75 \%$ relative humidity $(\mathrm{RH})$ for long period (three weeks). ${ }^{39)}$ In our study, sintering was conducted at $70^{\circ} \mathrm{C}$ for 15 min only. That is why we consider that the influence of sintering on the ibuprofen content is also minimum.

Effect of Sintering on Drug Release from Gum Tablets The release of drug from gum tablets was also investigated,
(A)

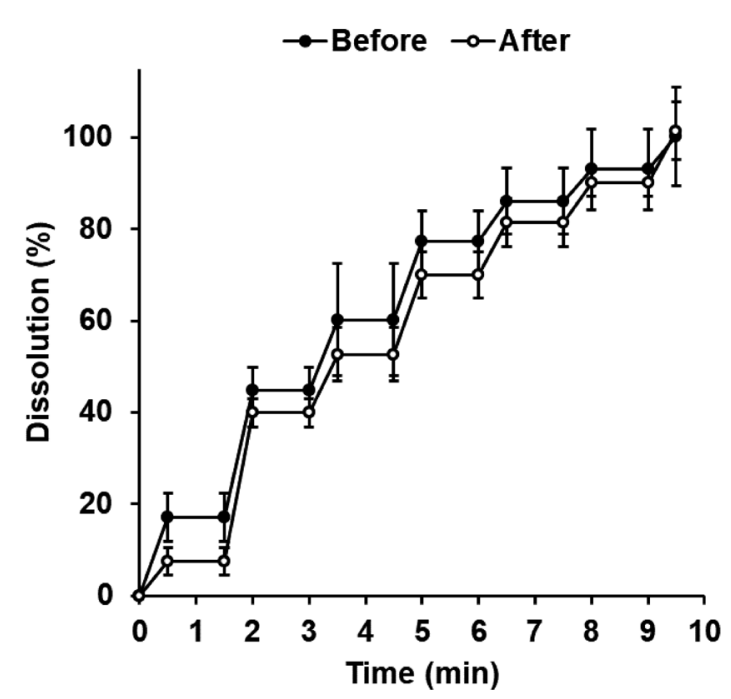

(B)

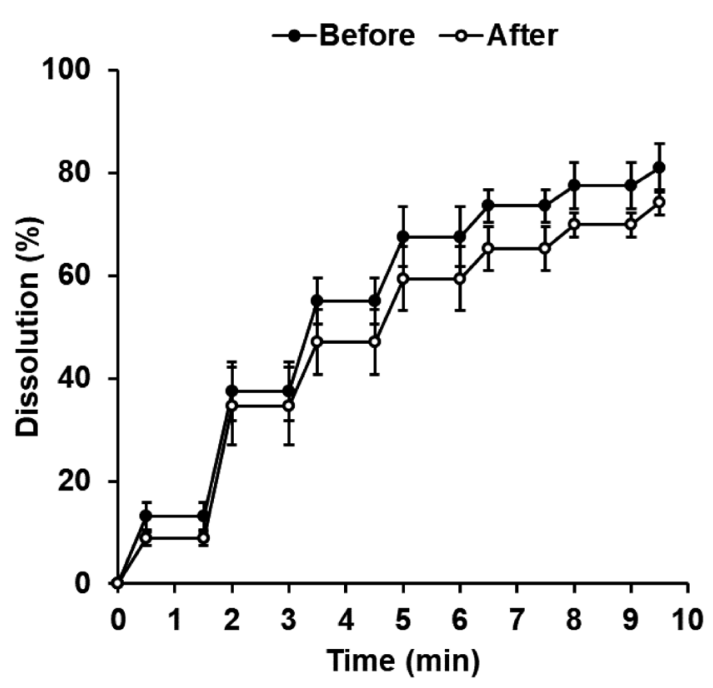

Fig. 6. Drug Release Profiles of (A) Ibuprofen-Loaded Gum Tablets and (B) Acetaminophen-Loaded Gum Tablets

Ibuprofen-loaded tablets or acetaminophen-loaded tablets were incubated at $70^{\circ} \mathrm{C}$ for $15 \mathrm{~min}$ or $80^{\circ} \mathrm{C}$ for $15 \mathrm{~min}$, respectively. The detailed method is described in Materials and Methods. Data represent the mean \pm S.D. $(n=3)$.

and the results are shown in Fig. 6. The release of drug components from MCGs differs considerably from the release from conventional oral formulations due to the chewing process. While a special apparatus for in vitro drug dissolution testing of MCGs has been proposed, ${ }^{40)}$ the equipment available for our study was limited. In the present study, we conducted in vitro chewing tests using semi-constant manual pressure applied via a plastic stick. The cycle of pressing and putting aside the gum tablets was repeated 7 times. A high proportion of both ibuprofen and acetaminophen was released from the gum tablets without heat treatment in only a few chewing cycles (ibuprofen-loaded tablets, $60.2 \pm 12.2 \%$; acetaminophenloaded tablets, $54.9 \pm 4.5 \%$; 3 cycles), and most of the ibuprofen and acetaminophen was ultimately released from the gum tablets (ibuprofen-loaded tablets, $100.3 \pm 10.8 \%$; acetaminophenloaded tablets, $80.9 \pm 4.7 \%$; 7 cycles).

Sintered ibuprofen-loaded and acetaminophen-loaded gum tablets both exhibited a delayed trend in drug dissolution (ibuprofen-loaded tablets, 52.6 $\pm 5.8 \%$; acetaminophen-loaded tablets, $47.1 \pm 6.3 \%$; 3 cycles) (Fig. 6). The sintering process 
reduces both the porosity and wettability of the tablets due to the fusion of granules. ${ }^{41}$ Rao et al. reported the drug release rate was inversely related with the sintering time of wax-based pellets. ${ }^{24)}$ Maboos et al. also reported extended drug release from sintered pellets composed of ethyl cellulose and wax. ${ }^{42)}$ These reports support our current drug release results.

Although we did not assess the bitterness-masking effect of gum tablets in the current study, we speculate that the bitterness of the drugs would be masked to some extent by the sweeteners (acesulfame K, sucralose) contained in the tablets. As several strong sweeteners (artificial sweeteners) are commercially available, the time to taste the sweetness varies (e.g., acesulfame $\mathrm{K}>$ sucrose $>$ sucralose). ${ }^{43}$ Combination of sweeteners can be effective for controlling the masking time. Suzuki et al. used various sweeteners (sucrose, xylitol, saccharin, saccharin sodium, aspartame, and sucralose) to mask the bitterness of acetaminophen in chewable tablets. ${ }^{44)}$ Based on these reports, we predict that the inclusion of artificial sweeteners into gum tablets would be useful in satisfying pediatric patients.

\section{CONCLUSION}

Confectionery xylitol gum-containing medical gum tablets were prepared and evaluated in this study. Sintering temperature and time directly affected the properties of both ibuprofen- and acetaminophen-loaded gum tablets. The hardness of the gum tablets was enhanced after appropriate sintering, and optimal sintering conditions were identified. The sintered gum tablets exhibited relatively extended drug release. Medical gum tablets may therefore be useful for pediatric patients or adult patients who can effectively chew gum. The gum tablets containing model drugs (acetaminophen and ibuprofen) may be applicable to remove pains derived from various kind of local and systemic diseases including cancer. Our data provide useful information to understand the preparation of xylitol gum-based drug tablets in the future clinical setting as a potential option in hospital pharmacies.

Acknowledgments The present research was partially funded by the OTC Self-Medication Promotion Foundation.

Author Contributions Tatsuaki Tagami: Conceptualization, Funding acquisition, Investigation, Methodology, Data curation, Supervision, Visualization, Writing-original draft, Writing-Review \& Editing, Project administration. Eriko Kuwata: Data curation, Formal analysis, Investigation, Visualization, Writing-original draft. Tetsuya Ozeki: Resources, Supervision.

Conflict of Interest The authors declare no conflict of interest.

\section{REFERENCES}

1) Aslani A, Rostami F. Medicated chewing gum, a novel drug delivery system. J. Res. Med. Sci., 20, 403-411 (2015).

2) Pagare PK, Satpute CS, Jadhav VM, Kadam V. Medicated chewing gum: A novel drug delivery system. J. Appl. Pharm. Sci., 2, 40-54 (2012).

3) Surana AS. Chewing gum: a friendly oral mucosa drug delivery system. Int. J. Pharm. Sci. Rev. Res., 4, 68-71 (2012).

4) Hartmann-Boyce J, Chepkin SC, Ye W, Bullen C, Lancaster T. Nicotine replacement therapy versus control for smoking cessation. Cochrane Database Syst. Rev., 5, CD000146 (2018).

5) Walsh RA. Over-the-counter nicotine replacement therapy: a methodological review of the evidence supporting its effectiveness. Drug Alcohol Rev., 27, 529-547 (2008).

6) Valoti M, Frosini M, Dragoni S, Fusi F, Sgaragli G. Pharmacokinetics of diphenhydramine in healthy volunteers with a dimenhydrinate $25 \mathrm{mg}$ chewing gum formulation. Methods Find. Exp. Clin. Pharmacol., 25, 377-381 (2003).

7) El Assassy AE, Amin MM, Abdelbary AA. Immediate release three-layered chewing gum tablets of fenoprofen calcium: preparation, optimization and bioavailability studies in healthy human volunteers. Drug Dev. Ind. Pharm., 38, 603-615 (2012).

8) Jójárt I, Kása P Jr, Kelemen A, Pintye-Hódi K. Study of the compressibility of chewing gum and its applicability as an oral drug delivery system. Pharm. Dev. Technol., 21, 321-327 (2016).

9) Mateti UV, Adla N, Rajakannan T, Valakkathala R. Insulin chewing gum: need of the day for diabetic patients. Int. J. Pharm. Investig., 1, 131-134 (2011)

10) Faraj JA, Dorati R, Schoubben A, Worthen D, Selmin F, Capan Y, Leung K, DeLuca PP. Development of a peptide-containing chewing gum as a sustained release antiplaque antimicrobial delivery system. AAPS PharmSciTech, 8, 26 (2007).

11) Behfarnia P, Aslani A, Jamshidian F, Noohi S. The efficacy of green tea chewing gum on gingival inflammation. J. Dent. (Shiraz), 17, 149-154 (2016).

12) Lingström P, Fure S, Dinitzen B, Fritzne C, Klefbom C, Birkhed D. The release of vitamin $\mathrm{C}$ from chewing gum and its effects on supragingival calculus formation. Eur. J. Oral Sci., 113, 20-27 (2005).

13) Imfeld T. Chlorhexidine-containing chewing gum. Clinical documentation. Schweiz. Monatsschr. Zahnmed., 116, 476-483 (2006).

14) Keukenmeester RS, Slot DE, Putt MS, Van der Weijden GA. The effect of medicated, sugar-free chewing gum on plaque and clinical parameters of gingival inflammation: a systematic review. Int. J. Dent. Hyg., 12, 2-16 (2014).

15) Bastian HL, Rindum J, Lindeberg H. A double-dummy, doubleblind, placebo-controlled phase III study comparing the efficacy and efficiency of miconazole chewing gum with a known drug (Brentan gel) and a placebo in patients with oral candidosis. Oral Surg. Oral Med. Oral Pathol. Oral Radiol. Endod., 98, 423-428 (2004).

16) Kimbrough $\mathrm{C}$, Chun $\mathrm{M}$, dela Roca G, Lau BH. PYCNOGENOL chewing gum minimizes gingival bleeding and plaque formation. Phytomedicine, 9, 410-413 (2002).

17) Aslani A, Ghannadi A, Raddanipour R. Design, formulation and evaluation of Aloe vera chewing gum. Adv. Biomed. Res., 4, 175 (2015).

18) Galande AD, Khurana NA, Mutalik S. Pediatric dosage formschallenges and recent developments: a critical review. J. Appl. Pharm. Sci., 10, 155-166 (2020).

19) Namiki N, Takagi N, Yuasa H, Kanaya Y. Studies on development of dosage forms for pediatric use (No. 1): preparation of gummi drug using gelatin (Japanese). Yakuzaigaku, 57, 86-94 (1997).

20) Tagami T, Ito E, Kida R, Hirose K, Noda T, Ozeki T. 3D printing of gummy drug formulations composed of gelatin and an HPMCbased hydrogel for pediatric use. Int. J. Pharm., 594, 120118 (2021).

21) Synaridou MS, Morichovitou AK, Markopoulou CK. Innovative pediatric formulations: ibuprofen in chocolate-coated honey core. $J$. Pharm. Innov., 15, 404-415 (2020).

22) Karavasili C, Gkaragkounis A, Moschakis T, Ritzoulis C, Fatouros DG. Pediatric-friendly chocolate-based dosage forms for the oral administration of both hydrophilic and lipophilic drugs fabricated with extrusion-based 3D printing. Eur. J. Pharm. Sci., 147, 105291 (2020)

23) Dave VS, Fahmy RM, Hoag SW. Near-infrared spectroscopic analysis 
of the breaking force of extended-release matrix tablets prepared by roller-compaction: influence of plasticizer levels and sintering temperature. Drug Dev. Ind. Pharm., 41, 898-905 (2015).

24) Rao M, Ranpise A, Borate S, Thanki K. Mechanistic evaluation of the effect of sintering on Compritol 888 ATO matrices. AAPS PharmSciTech, 10, 355-360 (2009).

25) Singh R, Poddar SS, Chivate A. Sintering of wax for controlling release from pellets. AAPS PharmSciTech, 8, E74 (2007).

26) Lin AY, Muhammad NA, Pope D, Augsburger LL. A study of the effects of curing and storage conditions on controlled release diphenhydramine $\mathrm{HCl}$ pellets coated with Eudragit NE30D. Pharm. Dev. Technol., 8, 277-287 (2003).

27) Desai KG, Schwendeman SP. Active self-healing encapsulation of vaccine antigens in PLGA microspheres. J. Control. Release, 165, 62-74 (2013)

28) Tanaka T, Kobayashi T, Takii H, Kamasaka H, Ohta N, Matsuo T, Yagi N, Kuriki T. Optimization of calcium concentration of saliva with phosphoryl oligosaccharides of calcium (POs-Ca) for enamel remineralization in vitro. Arch. Oral Biol., 58, 174-180 (2013).

29) Gazal G, Al-Samadani KH. Comparison of paracetamol, ibuprofen, and diclofenac potassium for pain relief following dental extractions and deep cavity preparations. Saudi Med. J., 38, 284-291 (2017).

30) Perioli L, Ambrogi V, Angelici F, Ricci M, Giovagnoli S, Capuccella M, Rossi C. Development of mucoadhesive patches for buccal administration of ibuprofen. J. Control. Release, 99, 73-82 (2004).

31) Liu Y, Sakurai K. Uniaxial negative thermal expansion of polyvinyl acetate thin film. Langmuir, 34, 11272-11280 (2018).

32) Jain H, Mulay S. A review on different modes and methods for yielding a pentose sugar: xylitol. Int. J. Food Sci. Nutr., 65, 135-143 (2014).

33) Bashyal S. Ibuprofen and its different analytical and manufacturing methods: a review. Asian J. Pharm. Clin. Res., 11, 25-29 (2018).

34) Panicker PS, Vigneshwaran LV, Bharathi MS. Formulation and evaluation of sintered matrix tablets of metformin hydrocloride.
Pharm. Sci. Monit., 8, 182-199 (2017).

35) Chen F, Wu JM, Wu HQ, Chen Y, Li CH, Shi YS. Microstructure and mechanical properties of 3Y-TZP dental ceramics fabricated by selective laser sintering combined with cold isostatic pressing. Int. J. Lightweight Mater. Manuf., 1, 239-245 (2018).

36) Sun Q, Nan C, Dai L, Xiong L. Effect of heat-moisture treatment with maltitol on physicochemical properties of wheat starch. Lebenson. Wiss. Technol., 62, 319-324 (2015).

37) Hadjikinova R, Marudova M. Thermal behaviour of confectionary sweeteners' blends. Bulg. Chem. Commun., 48, 446-450 (2016).

38) Lovett D, Tahir F. Hot melt extrusion technology for continuous manufacturing. European Pharmaceutical Review, 2018, 70561 (2018).

39) Cory WC, Harris C, Martinez S. Accelerated degradation of ibuprofen in tablets. Pharm. Dev. Technol., 15, 636-643 (2010).

40) Al Hagbani T, Nazzal S. Medicated Chewing Gums (MCGs): Composition, Production, and Mechanical Testing. AAPS PharmSciTech, 19, 2908-2920 (2018)

41) Bodke AR, Aher SS, Saudagar RB. A Review on SinteringTechnique in Pharmaceutical Sciences. Int. J. Curr. Pharm. Rev. Res., 8 , 130-133 (2017)

42) Maboos M, Yousuf RI, Shoaib MH, Nasiri I, Hussain T, Ahmed HF, Iffat W. Effect of lipid and cellulose based matrix former on the release of highly soluble drug from extruded/spheronized, sintered and compacted pellets. Lipids Health Dis., 17, 136 (2018).

43) Walsh J, Cram A, Woertz K, Breitkreutz J, Winzenburg G, Turner R, Tuleu C. Playing hide and seek with poorly tasting paediatric medicines: do not forget the excipients. Adv. Drug Deliv. Rev., 73, 14-33 (2014).

44) Suzuki H, Onishi H, Hisamatsu S, Masuda K, Takahashi Y, Iwata M, Machida Y. Acetaminophen-containing chewable tablets with suppressed bitterness and improved oral feeling. Int. J. Pharm., 278, 51-61 (2004). 